\title{
Mejora en el conocimiento hidrogeológico de la Masa de Agua Subterránea Jaizkibel
}

\author{
Jaizkibel Lurrazpiko Ur-Masaren ezagutza hidrogeologikoaren hobekuntza \\ Improvement of the knowledge about Jaizkibel Groundwater Body
}

\author{
Ane Zabaleta ${ }^{*}$, Iñaki Antiguedad ${ }^{1}$, Andoni da Silva², Inma Mugerza ${ }^{3}$, Patxi Tames ${ }^{2}$ \\ ${ }_{1}^{1}$ Hidrogeologia-Ingurumena Taldea, Geodinamika Saila, UPV-EHU, 48940 Leioa (Bizkaia). \\ ${ }^{2}$ Ingurumena eta Lurralde Antolaketa, Gipuzkoako Foru Aldundia, 20004 Donostia. \\ ${ }^{3}$ Hydrolur. \\ *Correspondencia: ane.zabaleta@ehu.es
}

\section{RESUMEN}

Este artículo actualiza el conocimiento hidrogeológico de la Masa de Agua Jaizkibel, integrando diversas técnicas de investigación utilizadas durante 2012 en un estudio promocionado por la Diputación de Gipuzkoa. Además del control piezométrico y de ensayos de bombeo, se han realizado diagrafías de conductividad eléctrica y temperatura en sondeos, parámetros que, con la carga hidráulica, han sido registrados en continuo en varios de ellos. También se ha actualizado el inventario de puntos de agua y efectuado análisis de aguas. La integración ha permitido mejorar el esquema hidrogeológico conceptual. Se trata de un sistema multicapa complejo, con alternancia de formaciones con carácter acuífero y otras actuando como acuitardos, lo que implica niveles productivos más o menos individualizados, como también reflejan los altos gradientes hidráulicos. Los ensayos han permitido estimar transmisividades de 20-60 m²/d y coeficientes de almacenamiento de 0,0004 y 0,0009, propios éstos de condiciones de cierto confinamiento. Se puede separar dos tipos de flujos: el profundo, del SO al NE, con aguas más mineralizadas, y con descarga tanto a surgencias como difusa al mar, y otro somero, asociado a zonas meteorizadas, con aguas de débil mineralización, y que drena a las numerosas regatas de la zona. Los bombeos no afectan al flujo de éstas, si bien hay que considerar su corta duración. Habrá que hacer un seguimiento riguroso de la explotación prevista en esta Masa para conocer su respuesta ante esas situaciones y precisar mejor los límites a establecer para evitar consecuencias no deseables en un ecosistema de indudable valor global.

PALABRAS CLAVE: Acuífero poroso, confinado, hidrogeología, multicapa.

\section{LABURPENA}

Artikulu honetan gaurkotu egiten da Jaizkibelgo Ur-Masaren ezagutza hidrogeologikoa. Asmo horrekin, Gipuzkoako Aldundiak sustatu eta 2012 urtean burutu den proiektuan erabilitako ikerketa-teknika desberdinen emaitzak integratu dira. Ohikoak diren kontrol piezometrikoa eta ponpaketasaioak egiteaz gain ez-ohikoak diren diagrafiak ere egin dira putzuetan, eroankortasun elektrikoa eta tenperatura sakonean zehar erregistratuz. Gainera, parametro biok, karga hidraulikoarekin batera, modu jarraian kontrolatu dira zenbait putzutan. Horretaz gain eguneratu egin da ur-puntuen inbentarioa eta uren analisiak egin ere. Datu guztien uztarketak lagundu egin du eskema hidrogeologiko kontzeptuala ontzen. Sistema multikapa konplexua dugu Ur-Masa hau, non akuifero portaera duten formazio batzuk akuitardo portaera duten beste batzuekin txandakatzen diren. Honen ondorioz akuiferoak bananduak agertzen dira, baina ez guztiz isolatuak. Hori ere gradiente hidrauliko altuek adierazia. Ponpa-saioetatik parametro nagusien balioak atera dira: transmisibitatea, 20 - 60 m²/egun, eta metatze-koefizientea, 0,0004 y 0,0009, azken hauek nolabaiteko konfinamendua jasaten duten akuiferoei dagozkienak direla. Bi ur-jario mota bereiz daitezke eskema kontzeptualean: alde batetik, sakonekoa, SWtik NErantz antolatua, mineralizatuagoak diren urekin, eta bai urbegi nagusietan eta bai modu lausoan itsasoan ere deskargatzen dena; eta bestetik, azalagoko urjarioa, azaleko zona meteorizatuagoei lotua, mineralizazio gutxiko urekin, eta inguruan ugari diren errekastoetan deskargatzen dena. Putzuetako ponpaketek ez dute errekastoetan eraginik sortu; hala ere, kontuan hartu behar da horien iraupen laburra. Aurrerantzean, Ur-Masa honetan aurreikusia den ustiaketaren gaineko segimendu zorrotza egin beharko da, ezagutu beharra dagoelako egoera horren aurrean Masako akuiferoek duten erantzuna. Ezagutza hau ezinbestekoa da ustiaketari jarri behar zaizkion mugak zehazteko, helburua izanik dudarik gabeko balio osoa duen Jaizkibelgo ekosisteman nahi ez den ondorioa ekiditea.

GAKO-HITZAK: Akuifero porotsua, geruza anitzekoa, hidrogeología, konfinatua

\section{ABSTRACT}

This paper updates the knowledge about the hydrogeology of the Jaizkibel Water Body. Several techniques were used during 2012 in a research work promoted by the Gipuzkoa Provincial Council: piezometric control, pumping tests, vertical measurement of electrical conductivity and temperature in the bore holes, and continuous measurements of electrical conductivity, temperature and hydraulic head in some of them. Additionally, the water point inventory was updated and water chemistry analyses were carried out. The integrated study of all the results allowed improving the conceptual hydrogeologic scheme. It is a multilayer complex system, with alternate aquifer and aquitard formations that imply the existence of individualized (in higher or lower degree) productive levels. This individualization is also reflected in the high hydraulic gradients observed. Transmisivity data $\left(20-60 \mathrm{~m}^{2} / \mathrm{d}\right)$ and data for storage coefficient 0,0004 y 0,0009, calculated from pumping tests are considered to be near from those of confined environments. Two types of fluxes can be distinguished: a deeper one, from SW towards springs or the sea in the NE, for which mineralization of waters is higher, and a shallower one, through weathered zones, with weakly mineralized waters that go to the numerous channels in the area. Pumping tests, do not affect runoff in channels, however, it is necessary to bear in mind that they are quite short. An exhaustive control of the foreseen exploitation in this Body has to be done in order to get to know its response to that kind of situation and to consciously precise the limits that have to be established to avoid non-desirable consequences in such a highly valuable ecosystem.

KEY WORDS: Confined, hydrogeology, porous aquifer, multilayer. 


\section{INTRODUCCIÓN}

El primer estudio hidrogeológico de Gipuzkoa data de 1971 y fue realizado por el Instituto Geológico y Minero de España. Entre sus conclusiones destaca que las aguas subterráneas no tienen una importancia significativa en los recursos hídricos del Territorio. Como consecuencia de estas conclusiones no se volvieron a realizar estudios de hidrogeología de cierta entidad hasta el "Estudio de evaluación de los recursos hidráulicos subterráneos del Territorio Histórico de Gipuzkoa", realizado en 1987 por el Servicio Geológico de Obras Públicas, del Ministerio de Obras Públicas, y la Diputación de Gipuzkoa. En dicho estudio se subdivide el Territorio en Unidades Hidrogeológicas, individualizándose la Unidad Cadena Costera, constituida por los materiales detríticos terciarios que se extienden desde Hondarribia hasta Zumaia. Dentro de este estudio, realizado según la metodología más utilizada en ese momento, se llevó a cabo cartografía hidrogeológica, inventario de puntos de agua con sus correspondientes aforos y balances de recursos, estudios hidroquímicos y, específicamente dentro de la unidad, se realizó un único sondeo con resultado negativo.

No es hasta el año 1993, en que por medio de un estudio realizado por la Diputación Foral de Gipuzkoa y el Ente Vasco de la Energía se continúa con la investigación hidrogeológica en la zona. En el año 1996 se edita por parte del Ente Vasco de la Energía el "Mapa Hidrogeológico del País Vasco, a escala 1:100.000" (EVE, 1996), donde se recopilan y sintetizan los conocimientos existentes hasta esa fecha.

En el año 1994 se habían realizado los primeros sondeos de investigación en el área del Jaizkibel. A partir de ese año se continua, por parte principalmente de la Diputación de Gipuzkoa y de la Dirección de Aguas del Gobierno Vasco, con los sondeos hidrogeológicos y las pruebas de bombeo, con el objetivo de tratar de regular los recursos hidráulicos subterráneos. En consecuencia, en 1999, el Ente Vasco de la Energía, por encargo de la Dirección de Aguas, elabora el informe "Balance de resultados de la investigación en la Unidad Hidrogeológica Jaizkibel (Gipuzkoa). Propuesta de explotación", así como, al año siguiente, el informe "Prueba de Producción Conjunta en los sondeos Jaizkibel-1,2,3,5,9 y DJH4 (Hondarribia-Gipuzkoa). Informe Final", donde se realizan las recomendaciones de explotación que traen como consecuencia las obras de captación y conducción de las tomas del Jaizkibel a la ETAP de Elordi en Irun, iniciadas en el año 2011 y finalizadas en el 2012.

En estos últimos años, se ha continuado con los estudios y los controles de caudales de manantiales y regatas, así como de niveles en los sondeos, por parte de la Diputación. En esos momentos, si bien se conocía el funcionamiento hidrogeológico general de la masa de agua, existían incógnitas sobre el funcionamiento hidráulico de los distintos sectores o acuíferos en que se compartimenta la misma. De forma que no se conocían las afecciones que pueden provocar unos bombeos continuados en el tiempo, y de cierta entidad, como los proyectados, en otros pozos y en los caudales de manantiales y regatas. Esto, unido a hecho de que la zona es Lugar de Interés Comunitario (LIC), y que próximamente va a publicarse, por parte de la Dirección de Biodiversidad del Gobierno Vasco, el documento "Objetivos y medidas de conservación para la designación de zona de especial conservación Jaizkibel (ES2120017)", ha propiciado que la Dirección de Medio Ambiente y Obras Hidráulicas de la Diputación Foral de Gipuzkoa haya promovido a lo largo del 2012 un estudio y una serie de controles foronómicos, piezométricos e hidroquímicos, con el objetivo de mejorar el conocimiento de la masa de agua subterránea, para poder realizar así, llegado el momento, una explotación de las captaciones compatible con el mantenimiento de los ecosistemas acuáticos del Jaizkibel.

Con este estudio, realizado por la empresa Hydrolur con el asesoramiento del Grupo de Hidrogeología y Medio Ambiente de la UPV-EHU, se ha querido profundizar en la dinámica de la masa de agua subterránea, mediante una interpretación conjunta de toda la información disponible, incluyendo, obviamente, toda la recopilada durante el estudio (actualización del inventario de puntos de agua, datos hidroquímicos, registros verticales en pozos, ensayos de bombeo, efecto de marea terrestre...). Especial atención se ha dado a la compartimentación de flujos subterráneos en la masa de agua, de gran trascendencia para la previsión de afección por parte de bombeos prolongados.

\section{MATERIAL Y MÉTODOS} Área de estudio

El área de estudio se localiza al NO del Territorio Histórico de Gipuzkoa, dentro de lo que se denomina como Masa de Agua Subterránea Jaizkibel. Aunque la Masa de Agua en su totalidad tiene una extensión de unos 26 km². el área estudiada se limita a la parte más nororiental, que se ubica entre la cuenca de la regata Artzu al este y la de Aierdi al oeste, ambas inclusive, y al norte de la divisoria de aguas superficiales (Fig. 1). Se trata de una zona con cotas que van desde el nivel del mar hasta superar los $500 \mathrm{~m}$ s.n.m., con pendientes medias del 20-25\%, y una red fluvial poco desarrollada, debido a la escasa distancia al mar y a la alta permeabilidad, en general, del sustrato rocoso. La precipitación media anual en esta zona es de unos 1700 mm, con una evapotranspiración anual media de 730 $\mathrm{mm}$; por tanto, se estima para toda la Masa de Agua de Jaizkibel una escorrentía total anual de $25 \mathrm{hm}^{3}$ y unos recursos medios subterráneos anuales de 13,8 hm³.

La Masa de Agua Subterránea Jaizkibel se encuentra formada en su totalidad por materiales del Terciario, que se disponen de manera concordante sobre los materiales del flysch detrítico-calcáreo del Cretácico Superior en una estructura monoclinal en la que localmente se observan pliegues suaves de orientación N40E a N60E y buzamientos de entre $10^{\circ}$ y $40^{\circ}$ hacia el NW. En la base de la serie estratigráfica se encuentra la Formación calcárea del Daniense, formada por margas, margocalizas y calizas de tonalidades rojas en la que las facies más carbonatadas llevan asociada una permeabilidad media. Por encima de ésta se posiciona 


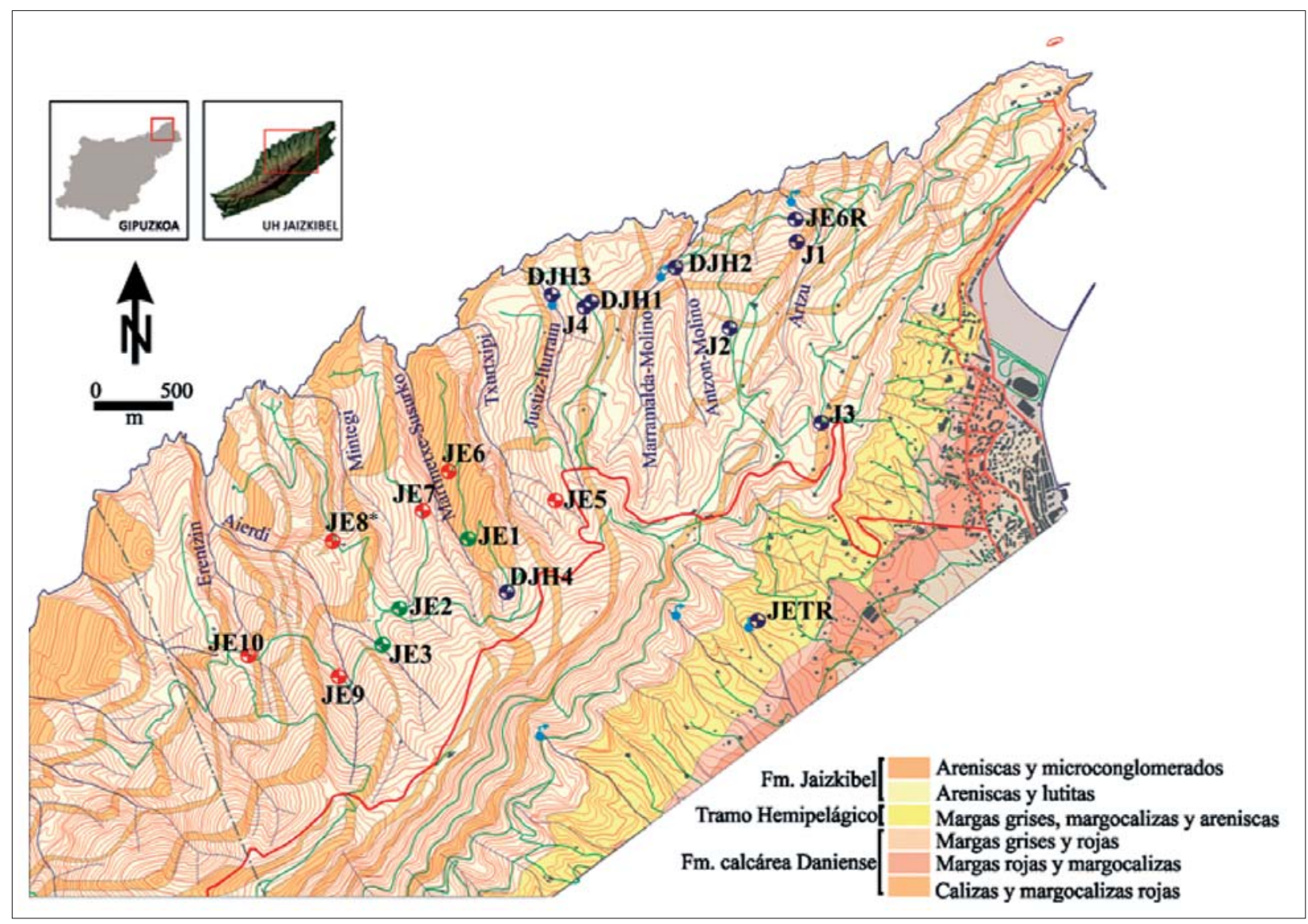

Fig. 1. - Masa de Agua Jaizkibel. Cartografía geológica. Divisoria de aguas (línea roja). Ubicación de los pozos y regatas. * El sondeo JE8 no se encuentra entubado. 1. Irudia. Jaizkibel Ur Masa. Kartografia geologikoa. Uren banalerroa (marra gorria). Putzuen eta erreken kokapena. * JE8 zundaketa entubatu gabe dago.

el tramo hemipelágico del Eoceno, que alcanza potencias de entre 250 y 300 m, y está representado, sobre todo, por margas; se le asigna una permeabilidad media-baja. A techo de la serie está la Formación Jaizkibel (Fig. 1), fundamentalmente terrígena con una potencia superior a los 1300 $\mathrm{m}$. Se trata del flysch detrítico del Terciario constituido, esencialmente, por la alternancia de un término de areniscas calcáreas, calizas arenosas y lutitas, y otro de areniscas, en ocasiones microconglomeráticas, estratificadas en bancos potentes, con frecuencia muy alteradas, y con un característico color amarillo. En estas últimas se centra el principal interés acuífero de la Masa de Agua.

La Masa de Agua Subterránea Jaizkibel no constituye un único acuífero. Se encuentra subdividida en varios acuíferos, más o menos individualizados, debido a la existencia de cambios laterales de facies a lutitas o a zonas de baja alteración-fracturación, de escasa permeabilidad. La formación más permeable, areniscas, posee una doble permeabilidad: primaria, por porosidad intergranular, y secundaria, por fisuración y disolución asociada; si bien en las perforaciones realizadas no se han evidenciado importantes procesos de karstificación en profundidad, sí que se encuentran zonas arenizadas, con mayor permeabilidad.

Se delimitan dos sistemas de flujo de sentido opuesto, cuyos límites deben coincidir, a grandes rasgos, con la di- visoria de aguas superficiales. Los dos manantiales principales (captados) que drenan la vertiente sur son Esteutz y Goikoerrota (Fig. 2). El primero se encuentra en el contacto entre la formación Jaizkibel y el sustrato margoso, pero el segundo aflora en medio de éste, seguramente a favor de la fracturación. La vertiente norte, a la que pertenece más del $80 \%$ de la superficie de afloramiento, es drenada, sobre todo, por las salidas al mar y por la surgencia Artzu y otras, también captadas, que vierten sus aguas a las regatas Errota y Justiz (Fig. 2). Los sondeos captados (JE1, JE2, JE3, JE5, JE9 y DJH4), junto con otros que se utilizan como puntos de observación, se encuentran en esta vertiente, en la que se ha centrado el presente estudio.

Según se recoge en el "Documento de objetivos y medidas de conservación para la designación de las zonas especiales de conservación Ulia ES2120014 y Jaizkibel ES2120017" (documento para aprobación definitiva, Ene. 2013), realizado por el Departamento de Política Territorial y Medio Ambiente del Gobierno Vasco, Jaizkibel presenta acantilados litorales con notables desniveles y acogen comunidades herbáceas y fruticosas características del medio salino y ventoso, junto con algunas especies de flora casmofítica y silicícola. Jaizkibel acoge numerosos microhábitats en los que se refugian especies extremadamente raras en la Comunidad Autónoma. 


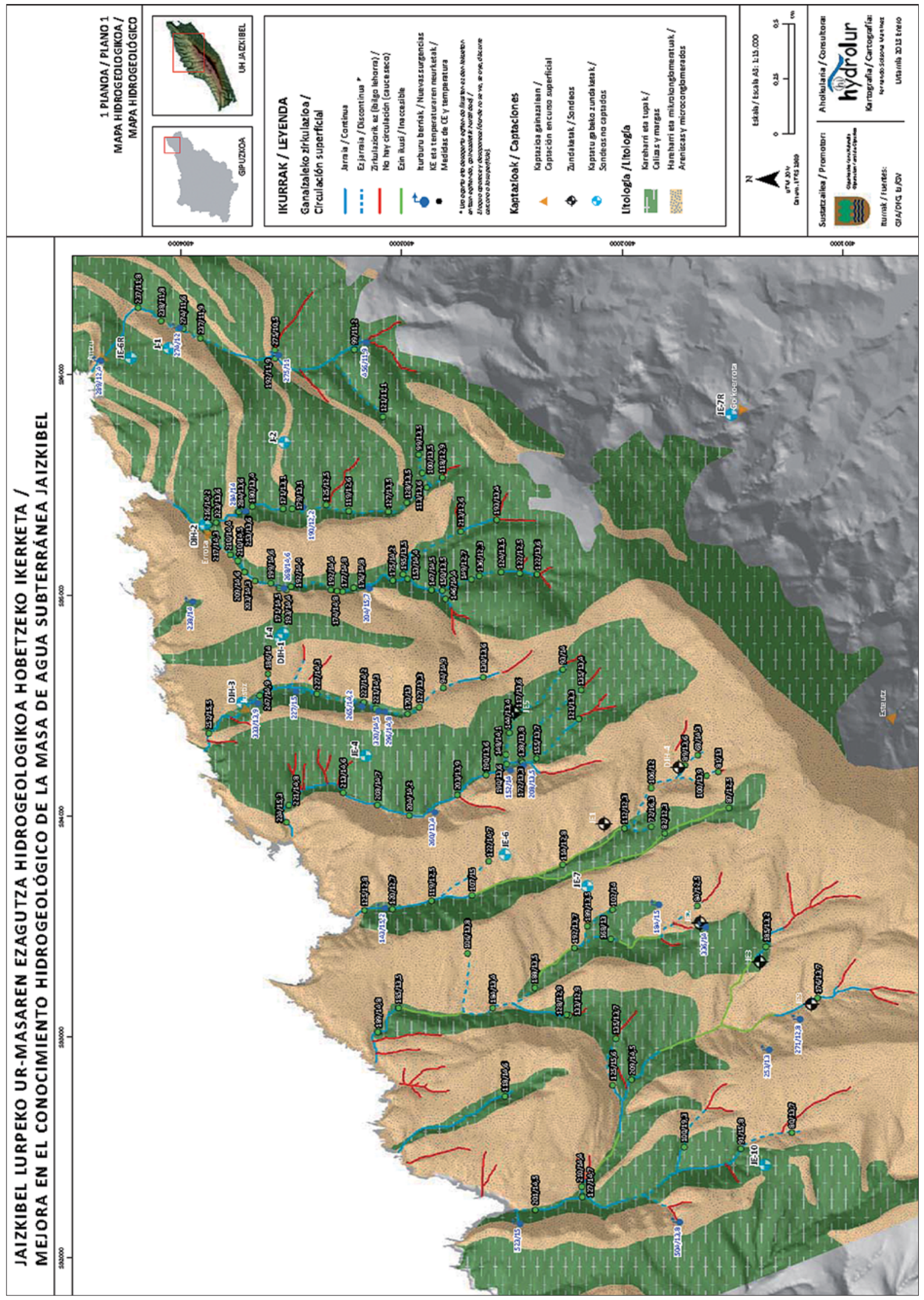

Fig. 2. - Mapa Hidrogeológico. Puntos de agua. Medidas de CE y Temperatura.

2. Irudia. Mapa hidrogeologikoa. Ur puntuak. Eroankortasun elektriko eta tenperatura neurketak. 


\section{Metodología}

El presente estudio se ha realizado a lo largo del 2012 en colaboración entre la Dirección de Medio Ambiente y Obras Hidráulicas de la Diputación Foral de Gipuzkoa, el Grupo de Hidrogeología (Universidad del País Vasco - Euskal Herriko Unibertsitatea) y la empresa Hydrolur. Durante este periodo se han utilizado varias técnicas para profundizar en el funcionamiento de la Masa de Agua Subterránea de Jaizkibel. Algunas son habituales en la investigación hidrogeológica, pero otras no lo son tanto. Se ha tratado de buscar la integración de todas ellas.

Se ha mejorado la cartografía geológica existente, ajustando los límites entre los contactos litológicos (Fig. 2). Para ello han sido de gran ayuda la ortofotografía, por un lado, y el trabajo de campo, por otro. Durante el trabajo de campo, y sobre todo entre los meses de May. y Jun. de 2012, se ha actualizado, además, el inventario previo de puntos de agua, en especial, a lo largo de las regatas (Artzu, Antzon-Molino, Marramalda-Molino, Justiz-Iturrain, Txurtxipi, Martitxene-Susurko, Mintegi, Aierdi y Erentzin; Fig. 1). La mayor parte de los puntos de agua identificados se localizan en los contactos entre diferentes litologías. En esta actualización se han tomado medidas in situ de la conductividad eléctrica (CE) y la temperatura del agua (Fig. 2), que proporcionan información sobre sus características, lo que permite, a posteriori, su consideración en el esquema conceptual de flujos de la Masa estudiada.

También se han realizado controles en algunos de los pozos existentes en la zona, más allá del habitual seguimiento temporal de la piezometría. Por un lado, con dispositivos de registro continuo (tipo Diver-CTD) de presión, temperatura y conductividad eléctrica (CE) en los pozos JE7, JE6, JE5, JE4, J2, DJH4 y DJH3, y de presión atmosférica en JE4, JE5 y DJH4. La medida de presión atmosférica es necesaria para conocer en cada momento la posición del nivel de agua de cada pozo. Todos los dispositivos se programaron para registrar una medida cada diez minutos. De esta forma, es posible observar la respuesta de los pozos a las precipitaciones y la evolución de los niveles piezométricos en su ausencia. Las medidas de CE y temperatura, referidas a la profundidad de colocación del dispositivo en la columna de agua, permiten conocer, en su caso, los cambios en el tipo de agua ahí presente. Hay que señalar que los dispositivos se han colocado coincidiendo con tramos ranurados en la entubación de los pozos, es decir, en zonas de flujo previsible de agua.

Por otro lado, y con el objetivo de analizar cambios que pueden darse en la vertical de la columna de agua, en los pozos donde ha sido posible introducir la sonda se efectuaron diagrafías (registros verticales de CE y temperatura), entre 1 y 3 veces por pozo, entre May. y Sep. 2012. El objetivo era observar, en su caso, las variaciones registradas en los pozos y explicarlas por el flujo entre los diferentes niveles permeables atravesados, llevando esta interpretación al esquema conceptual de funcionamiento de la Masa de Agua.

Se han seguido haciendo aforos, como se hace desde 2010, en las regatas Aierdi, Antzon-Molino, Artzu, Erentzin, Justiz-Iturrain, Marramalda-Molino 2, Martitxene-Susurko,
Mintegi y Txurtxipi y en el manantial de Artzu. Además, se han recopilado los análisis hidroquímicos existentes desde 1997, tanto de regatas como de pozos, y se ha realizado un tratamiento actualizado de los datos analíticos, estableciendo relaciones entre iones y aguas, y con una interpretación de la información en términos hidrogeológicos. Es claro que las aguas de esta Masa son de buena calidad, por eso el estudio hidroquímico realizado se interesa por el contexto hidrogeológico en el que las aguas adquieren sus características.

Por último, entre el 24.09.2012 y 01.10.2012 se realizaron ensayos de bombeo en coordinación con servicios de Txingudi. En un principio se realizaron ensayos individuales, de 23 horas de duración cada uno, en los sondeos JE9, JE1, JE3, DJH4, JE2 y JE5, respectivamente, a excepción del bombeo en el pozo JE5 que se alargó durante más de dos días. Todos estos pozos se localizan en el sector SO del área objeto de estudio (Fig. 1). En estos ensayos se bombearon entre 15 y 20 l/s por pozo, a excepción del JE2 (8 l/s) y del JE5 (35 l/s).

Tras la finalización de los bombeos individuales se dejó un tiempo de recuperación de 24 h y el 02.10.2012 se procedió a realizar un ensayo de bombeo conjunto durante una semana. Este ensayo supuso un caudal total de extracción de $63 \mathrm{l} / \mathrm{s}$, sin contar el del JE5 que fue variable por problemas con la bomba. De estos ensayos se calcularon los valores de los parámetros hidrogeológicos básicos de un acuífero, transmisividad $(\mathrm{T})$ y coeficiente de almacenamiento (S), y se analizaron las relaciones entre unos pozos y otros. Al inicio y al finalizar el conjunto de ensayos de bombeo se realizaron muestreos de agua en los pozos para su análisis, y se realizaron aforos en las regatas y manantiales antes mencionadas para evidenciar el posible efecto de los bombeos sobre los mismos.

\section{RESULTADOS Y DISCUSIÓN}

El trabajo de campo ha permitido ajustar los límites litológicos en algunas zonas del área de estudio, principalmente en el sudoeste (entre los sondeos JE3 y JE10). Lo abrupto del terreno y la densa maleza ha dificultado el trabajo en algunas zonas, en las que se ha utilizado la ortofotografía para, relacionando las diferentes litologías con e relieve del entorno (resaltes, vaguadas...), localizar con un cierto detalle los contactos entre materiales. La actualización del inventario de puntos de aguas también ha sido importante, ya que, además de haber permitido situar nuevos aportes de agua, muchos de esos puntos se asocian a contactos entre materiales de diferente permeabilidad. La Fig. 2 recoge los puntos de agua (surgencias) encontrados.

En todos los arroyos de la vertiente norte, la conductividad eléctrica (CE) de las aguas es baja en cabecera y va aumentando en el sentido de la corriente a medida que se van añadiendo nuevos aportes, normalmente de naturaleza difusa, de agua más mineralizada. Así, en cabecera, donde el caudal es muy escaso en situaciones de estiaje $(<1 \mathrm{l} / \mathrm{s})$, incluso nulo en algunas regatas, las aguas presentan valores de CE habitualmente inferiores a 130 $\mu \mathrm{S} / \mathrm{cm}$, indicando una muy baja mineralización. Aguas 
abajo, sin embargo, aumenta de forma progresiva, alcanzando en las partes bajas valores de 200-250 $\mu \mathrm{S} / \mathrm{cm}$, con caudales que en esa situación de estiaje pueden llegar a la docena de I/s. Se trata de aguas de facies bicarbonatada cálcica, como corresponde a la litología carbonatada de los materiales allí presentes. En cualquier caso, estos valores de CE de las aguas de las regatas están bastante por debajo de los encontrados en las aguas de los pozos (hasta los $430 \mu \mathrm{S} / \mathrm{cm}$ ), lo que muestra, como punto de partida, una evidente diferenciación de flujos, siendo los más someros los que descargan a las regatas del entorno. El aumento de la mineralización en la parte baja de las regatas, sobre todo en los manantiales allí presentes (290 $\mu \mathrm{S} / \mathrm{cm}$ en Artzu, $330 \mu \mathrm{S} / \mathrm{cm}$ en Justiz) hace pensar en una cierta descarga de los flujos más profundos en esas zonas, produciéndose mezcla con los aportes más someros.

El control de niveles llevado a cabo en los pozos evidencia, por un lado, que, sin bombeos, en un mismo pozo el nivel piezométrico tiene variaciones escasas, inferiores a 1 metro, y, por otro lado, que las diferencias entre las cotas piezométricas de los pozos son grandes (Fig. 3). Como ejemplo, el nivel en DJH4 se encuentra a una cota próxima a los $220 \mathrm{~m}$, mientras que en JE4 está en torno a los $60 \mathrm{~m}$. La distancia en línea recta entre ambos es de 1409 m, por lo que el gradiente hidráulico sería de un $11 \%$, lo que hace pensar en una desconexión hidráulica entre ellos, o bien en la existencia de materiales de baja permeabilidad (acuitardos) entre ambos. De hecho, como se ha comentado con antelación, en el caso de la Masa de Agua Subterránea Jaizkibel no estaríamos hablando de un acuífero extenso y potente, sino de un conjunto de niveles acuíferos, más o menos individualizados, asociados a las formaciones más productivas (areniscas, con pasadas de microconglome- rados), y separados por formaciones que actúan como acuitardos (areniscas calcáreas, calizas arenosas y lutitas). Se trataría, en conjunto, de un sistema acuífero multicapa, con la complejidad que ello lleva asociada.

El orden de los pozos, de mayor a menor cota piezométrica, considerando todos los datos disponibles, es (ver ubicación de los pozos en la figura 1): JE9 (233 m, surgente), DJH4 (223 m), JE10 (204 m), JE1 (196 m), JE2 (194 m), JE7 (161 m), JE5 (117 m), JE6 (81 m), J2 (77 m), DJH1 (66 m), J4 (60 m), JE4 (58 m), J1 (47 m), DJH3 (33 m). Esta distribución de cargas hidráulicas define un flujo subterráneo desde el suroeste (JE9, DJH4, JE10) hacia el noreste (J1, DJH3), donde se localizan las surgencias de Artzu y Justiz. Ya anteriormente se ha comentado que estas surgencias deben ser lugar de una cierta descarga de los flujos más profundos, junto con aportes más superficiales, habida cuenta de la mineralización de sus aguas. De hecho, la mineralización de las aguas profundas va incrementándose en el sentido del flujo, desde valores del orden de 180-250 $\mu \mathrm{S} / \mathrm{cm}$ (DJH4, JE9, JE3) hasta 400-430 $\mu \mathrm{S} / \mathrm{cm}(\mathrm{JE} 4, \mathrm{DJH} 1, \mathrm{DJH3}, \mathrm{J} 2)$.

Las lecturas diezminutales de nivel en los pozos JE7, JE6, JE5, JE4, J2, DJH4 y DJH3 han aportado información de gran interés. Destaca el hecho generalizado de la muy escasa respuesta a la precipitación por parte de los niveles piezométricos de los pozos (Fig. 3), lo cual es indicativo de la dificultad de acceso del agua de lluvia a la zona saturada de los niveles atravesados por los pozos. Hablamos, por tanto, de una recarga lenta y difusa, sin la existencia de flujos concentrados a través de áreas preferenciales. Las formas de erosión-disolución observables en superficie no tienen continuación en profundidad, al menos de forma organizada para el sistema de flujo actual.

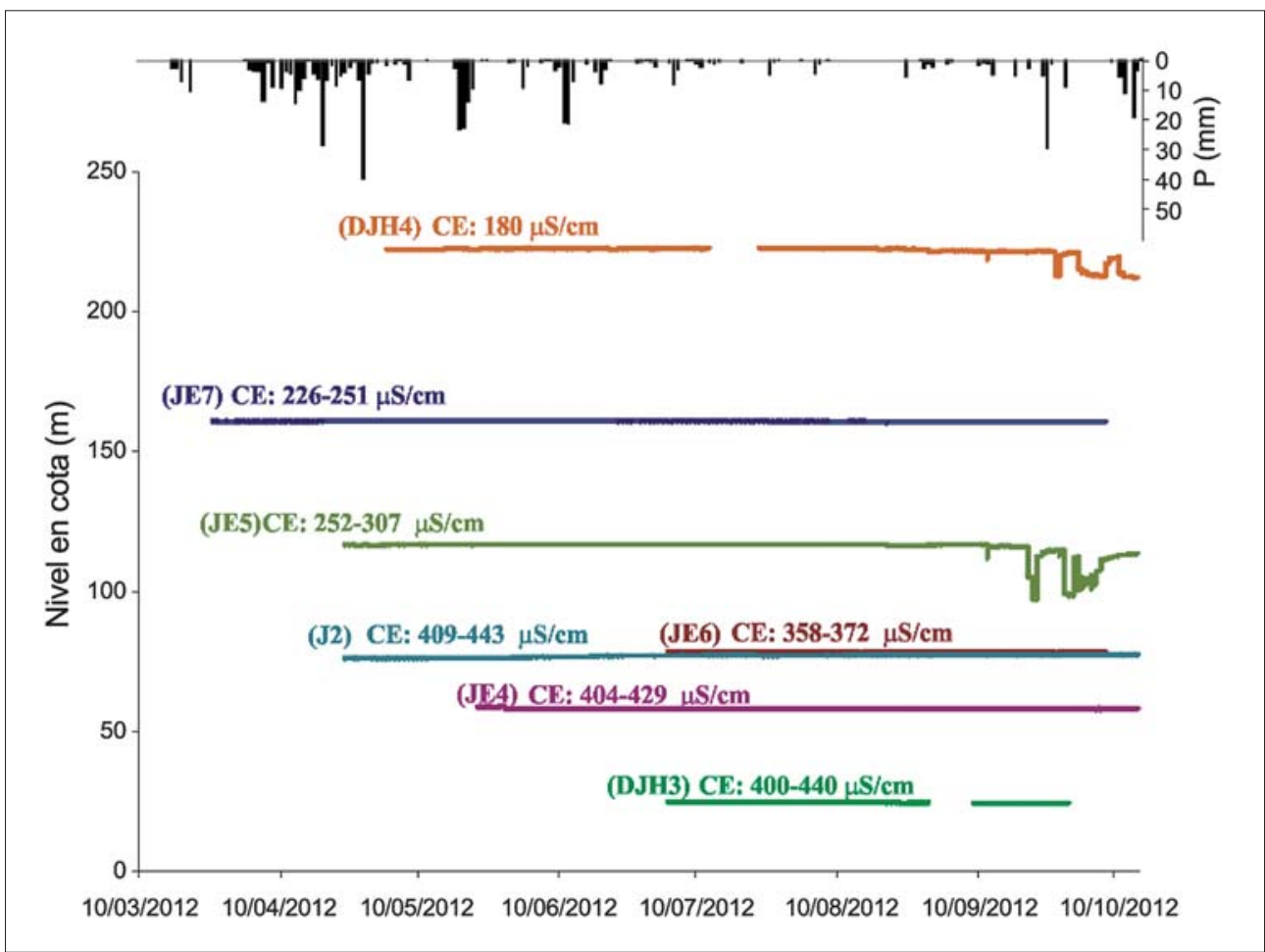

Fig. 3. - Evolución piezométrica en los pozos con registro continuo. Valores de CE en los pozos. Precipitaciones diarias. 3. Irudia. Ur-mailaren eboluzioa kontrol jarraia duten putzuetan Putzuetako eroankortasun elek triko balioak. Eguneko prezipitazioak. 
El registro continuo de niveles ha permitido, también, observar pequeñas variaciones en las aguas de algunos sondeos como consecuencia de los ensayos de bombeo llevados a cabo a finales de Sep. y principios de Oct. de 2012. En general, los bombeos individuales (de prácticamente 1 día de duración) apenas causan variación del nivel piezométrico en los pozos controlados en el entorno; sólo en el caso del JE4 se llega a observar un ligero descenso, unos $15 \mathrm{~cm}$, cuando se bombea JE5 (el de mayor caudal, $35 \mathrm{l} / \mathrm{s}$, y duración, 2 días), lo que indicaría una mejor conexión hidráulica entre ambos pozos. Cuando se realiza el bombeo conjunto se observa un ligero descenso, unos $10 \mathrm{~cm}$, en JE4 y JE7, situados relativamente cerca de los pozos bombeados, pero ningún descenso en los más alejados (J2, DJH3), situados cerca de las surgencias (Justiz, Errota, Artzu; Fig. 1 y 2). Tampoco se han observado variaciones en los caudales de las regatas y manantiales de la zona durante este bombeo conjunto, lo que evidencia que se trata de flujos diferenciados.

Por otro lado, el control continuo ha puesto en evidencia fluctuaciones diarias de nivel del orden centimétrico. Este hecho se ha constatado en casi todos los sondeos monitorizados, aunque en algunos resulta más evidente, tal es el caso de J2, en el que puede sobrepasar amplitudes de $30 \mathrm{~cm}$ (Fig. 4). En el resto, la amplitud media oscila entre 3 y $8 \mathrm{~cm}$, alcanzando los $15 \mathrm{~cm}$ en algunas épocas. Los máximos y mínimos no se alcanzan a la vez en todos los sondeos, existiendo desfases entre 0,5 y 2 horas. En un principio, la existencia de dos máximos y dos mínimos diarios, junto con la proximidad de la Masa de Agua a la costa, condujo a pensar en la influencia mareal directa en los niveles piezométricos. Es de sobra conocido el efecto de las mareas oceánicas sobre acuíferos confinados cercanos a la costa, como consecuencia de una variación cíclica de la carga de la masa de agua oceánica sobre el acuífero. Además, las fluctuaciones observadas en Jaizkibel se relacionan muy bien con los máximos y mínimos para el puerto de Pasaia, como se observa en la figura.

Sin embargo, siguiendo el método de Ferris (1951), que relaciona la amplitud de las fluctuaciones que se ob- servarían en un sondeo a partir de los parámetros hidráulicos del acuífero (T y S) y las características de las propias mareas oceánicas, se evidenció que la posible influencia de la marea oceánica sobre las fluctuaciones observadas en Jaizkibel es mínima. Por tanto, hay que pensar que esas fluctuaciones deben tener su principal origen en las mareas terrestres. Este tipo de afección (Bredehoeft, 1967), debida a la dilatación de la Tierra (las fuerzas de gravedad que provocan las mareas oceánicas también deforman la corteza terrestre), es también de sobra conocido en la literatura científica, en el caso de acuíferos confinados, y en nuestro entorno ha sido puesto de manifiesto en acuíferos alejados de la costa, como en la Unidad Hidrogeológica de las Calizas de Subijana en Álava-Araba (Fernández de Ortega, 2007). En cualquier caso, es ésta una cuestión en la que hay que profundizar, a partir del estudio de registros de mayor longitud temporal.

Además, se han realizado trece diagrafías en los sondeos equipados con los dispositivos Diver-CTD. La mayor parte de ellas se realizaron en May. y Ago. de 2012. A la hora de analizar esos registros verticales es muy importante conocer el acabado de los pozos, es decir, la posición de los tramos de tubería ranurada, ya que son los tramos de acceso del agua al pozo. Así, las observaciones pueden mostrar, en su caso, flujo vertical dentro del propio pozo; por ejemplo, entre diferentes niveles productivos atravesados por el mismo. Las diagrafías efectuadas muestran registros muy similares para cada pozo, sin importar el momento del registro. Esto indica, como se venía pensando por el resto de observaciones, que el flujo en las formaciones permeables del Jaizkibel es de tipo difuso, inercial, sin grandes alteraciones.

A modo de ejemplo, la figura 5 muestra los tres registros de CE y temperatura realizados en el pozo JE5 (24.04.2012, 04.05.2012 y 30.08.2012), junto con la columna litológica atravesada y el acabado del pozo. En este caso, los tramos abiertos de la entubación se sitúan en la parte baja, en la formación más permeable (areniscas, fundamentalmente). La temperatura se mantiene constante durante los primeros $20 \mathrm{~m}$ y a partir de ahí, a medida que se

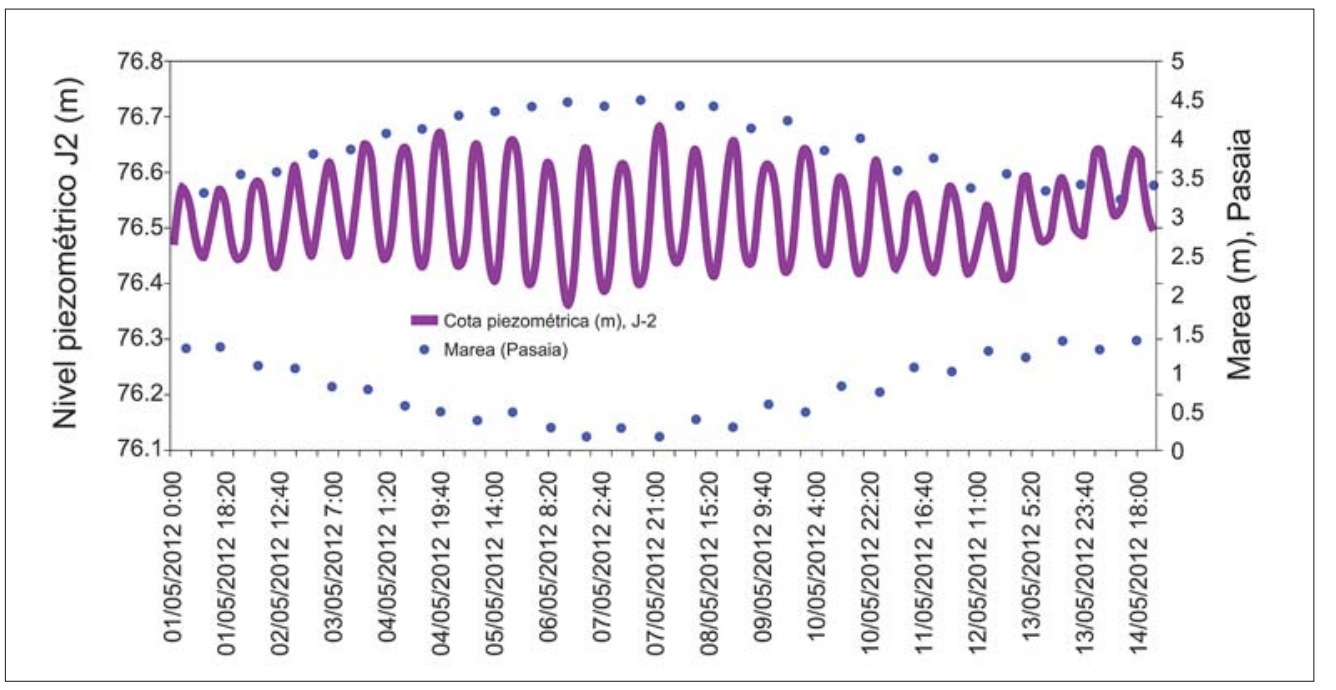

Fig. 4. - Evolución del nivel piezométrico en $J$ 2 versus altura de la marea en Pasaia.

4. Irudia. J-2ko maila piezometrikoaren eboluzioa versus Pasaiako marearen altuera. 


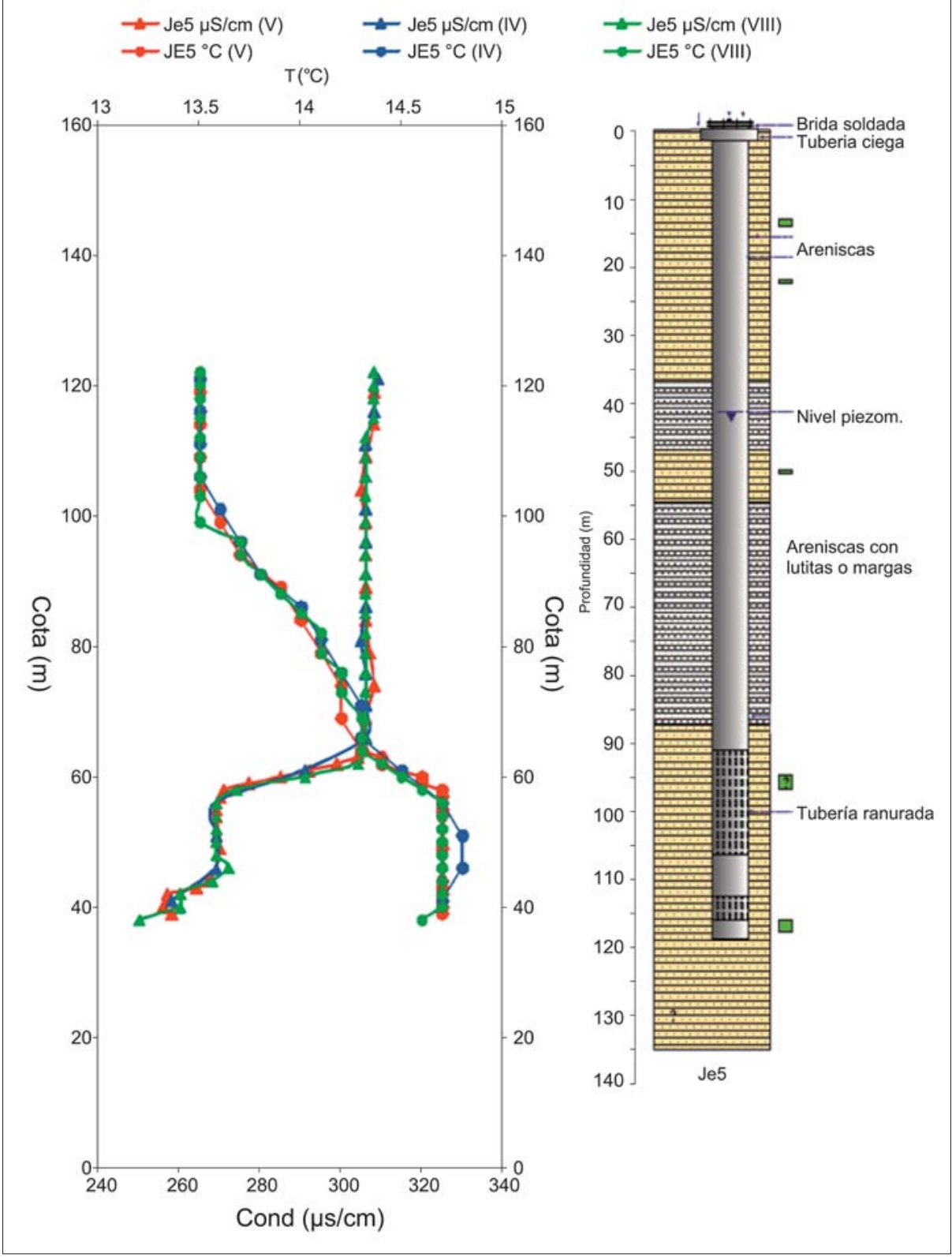

Fig. 5. - Diagrafías realizadas en el pozo JE5 entre Abr. y Ago. 2012. 5. Irudia. JE5 putzuan 2012ko Api. eta Abu. artean egindako diagrafiak. desciende en el sondeo, empieza a ascender de forma más o menos constante, pasando en $40 \mathrm{~m}$ de 13,5 a $14,7^{\circ} \mathrm{C}$ (gradiente: $3^{\circ} \mathrm{C} / 100 \mathrm{~m}$ ). Es ahí donde se encuentra la zona ranurada superior. A partir de ese tramo la temperatura se mantiene de nuevo constante. La CE se mantiene invariable, sobre $305 \mu \mathrm{S} / \mathrm{cm}$, hasta la zona ranurada, lugar en el que experimenta un importante descenso, hasta los $270 \mu \mathrm{S} / \mathrm{cm}$; ésta es el agua que entra al sondeo, y a la altura de la segunda zona ranurada vuelve a descender hasta $250 \mu \mathrm{S} / \mathrm{cm}$.

La columna de agua hasta la profundidad del tramo ranurado está prácticamente estancada, poco renovable. Esto justificaría el mantenimiento de los valores de CE, con aguas que deben de corresponder a entradas antiguas. Las aguas que en los momentos de las diagrafías atraviesan el sondeo por alguno de los tramos ranurados son aguas de menor mineralización (CE del orden de 260-270 $\mu \mathrm{S} / \mathrm{cm})$ que, en parte, se mezclan con las previas generando los saltos observados. El mantenimiento de la temperatura en los primeros $20 \mathrm{~m}$ de la columna podría explicarse por el efecto regulador del aire en la parte superior del sondeo. Su descenso entre 100 y $60 \mathrm{~m}$ da un gradiente de $3^{\circ} \mathrm{C} / 100 \mathrm{~m}$, que podría ser una aproximación al gradiente geotérmico de esta zona geográfica. En otros pozos (JE6, JE7) las diagrafías indican un flujo vertical en el interior del pozo, entre tramos abiertos a distinta profundidad, lo que evidencia que los propios sondeos pueden conectar distintos niveles acuíferos si tienen tubería ranurada en ellos. Esto hace que haya que interpretar con precaución los registros, y que éstos cubran diferentes situaciones hidrológicas, para poder extraer conclusiones precisas.

Entre el 24.09.2012 y 10.10.2012 se realizan ensayos de bombeo. Los valores de transmisividad (T) obtenidos, 
mediante los métodos de interpretación habituales (Theis, Jacob, Hantush), presentan cierta variación espacial. JE9, JE3, JE5 y DJH4 muestran una T similar (en torno a 60 $\mathrm{m}^{2} / \mathrm{d}$ ), aunque en el primero desciende a la mitad por el efecto de una barrera negativa, que puede relacionarse con un límite impermeable cercano. En el sondeo JE2 la T es del orden de $20 \mathrm{~m}^{2} / \mathrm{d}$ y en JE1 próxima a $30 \mathrm{~m}^{2} / \mathrm{d}$. Esta variación espacial en los valores de T es acorde con el esquema de acuífero multicapa que estamos considerando para esta Masa de Agua. Es, además, coherente con una cierta heterogeneidad espacial de la función transmisiva de las diferentes formaciones acuíferas.

Junto al sondeo JE5 y al sondeo DJH4 existen sendos sondeos (JE5 bis y DJH4 bis), a una distancia de unos 20 $\mathrm{m}$, que se han utilizado como pozos de observación para extraer más información de los ensayos. Esto ha permitido calcular el Coeficiente de Almacenamiento (S), indicativo de la función capacitiva de un acuífero. Los valores

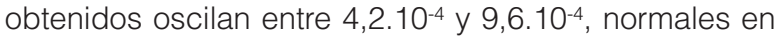
acuíferos confinados 0 , incluso, semi-confinados, lo que también es acorde con el esquema conceptual considerado. La aproximación de Hantush, para acuíferos semiconfinados, permite estimar la permeabilidad vertical del acuitardo ( $K^{\prime}$ ) en 0,0034 y 0,0075 m/d, para el caso de esos dos sondeos (DJH4, JE5). Este valor, a pesar de las incertidumbres asociadas, es indicativo del papel que pueden estar jugando los acuitardos, como transmisores de agua, entre los acuíferos del sistema multicapa que supone esta Masa de Agua.

Ya anteriormente se ha hecho mención a la práctica inexistencia de afección desde los pozos bombeados a los pozos del entorno; sólo en algún caso se ha observado un descenso (el caso citado del bombeo en JE5 sobre el JE4) del orden de $15 \mathrm{~cm}$. De todas maneras, hay que tener muy presente que la duración de los ensayos de bombeo (casi 1 día en el caso de los individuales), si bien permite el cálculo de los parámetros hidrogeológicos básicos (T, S), no permite, sin embargo, evidenciar los efectos que bombeos más prolongados, en condiciones de explotación, pudieran tener en el entorno. Lo que sí muestran los ensayos efectuados es la no existencia de descensos claros en los sondeos del entorno. A la vista de los datos de T y S calculados, los radios de influencia esperables en los ensayos de esa duración están entre 200 y 500 m, lo que explicaría lo observado.

La Fig. 6 muestra la variación de la cota piezométrica (m) y de la conductividad eléctrica (CE, $\mu \mathrm{S} / \mathrm{cm}$ ) registrada en el pozo JE5 en un periodo de tiempo que incluye un bombeo en él realizado. Sirve como ejemplo del interés que este seguimiento detallado ofrece para ir aclarando la complejidad del esquema de flujos, en este caso en condiciones de bombeo. Este produce un descenso del nivel de unos $18 \mathrm{~m}$ en el propio pozo bombeado, nivel que se recupera de forma inmediata una vez finalizado el bombeo, lo que evidencia la capacidad del acuífero afectado para suministrar el caudal demandado durante el tiempo de bombeo. Sin embargo, la figura indica que no siempre es el mismo tipo de agua la que está en el pozo. Esta presenta variaciones súbitas significativas de CE, entre 260 y $300 \mu \mathrm{S} / \mathrm{cm}$; también la temperatura muestra variaciones similares, no mostradas en la figura. Los cambios observados son consecuencia del efecto del bombeo, que moviliza tanto el agua de los niveles productivos asociados como la presente en el pozo antes del bombeo (Fig. 5). Obviamente, la interpretación de este tipo de gráficas es específica para cada sondeo, por lo que hace falta series de datos más largas, en condiciones diferentes, y en más pozos para poder extraer conclusiones con valor para el conjunto multicapa de la Masa de Agua.

Por otro lado, justo antes del inicio y justo después del final de los ensayos de bombeo se muestrearon las aguas de los sondeos para su posterior analítica. La primera de ellas la realizó Servicios de Txingudi el día 12.09.2012, y la segunda la Diputación Foral el 08.10.2012. Los resultados analíticos muestran que los bombeos han producido algunos cambios en la conductividad eléctrica de las aguas, aumentando ésta en la mayoría de los pozos (JE1, JE3, JE9, DJH4), pero disminuyendo en otros (JE2 y JE10). Estos cambios, si bien de escasa entidad, son indicativos de una cierta movilización de agua entre los pozos por efecto de los bombeos, aunque ésta no haya sido suficiente para provocar descensos sustanciales en el nivel piezométrico.

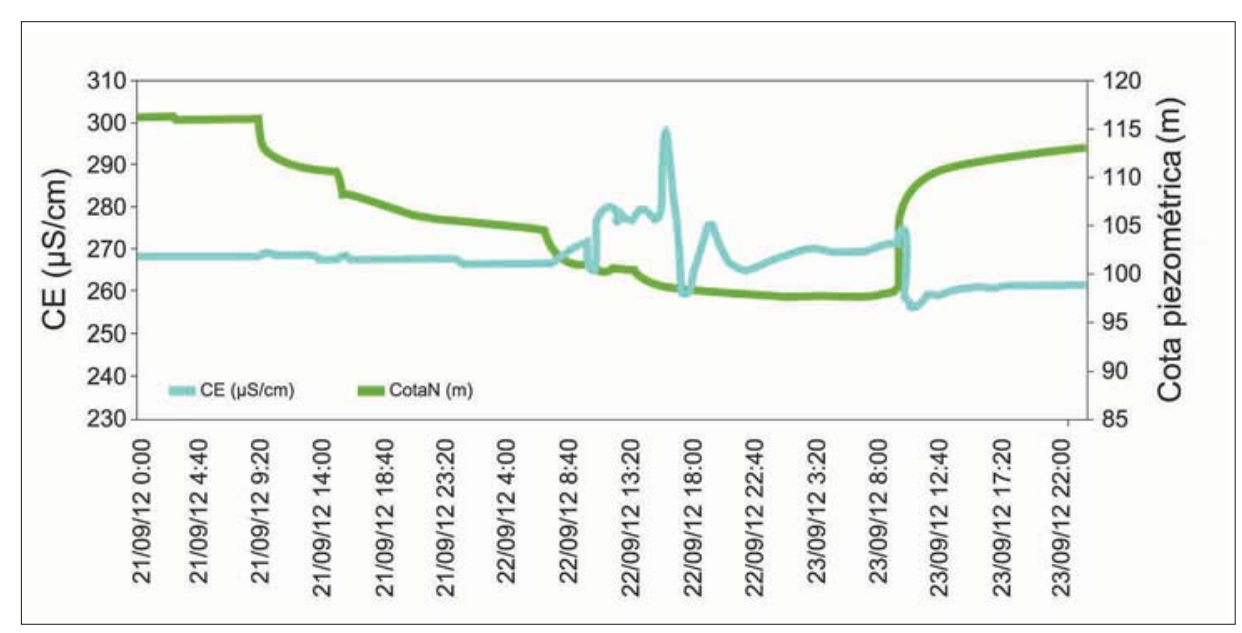

Fig. 6. - Variación de la cota piezométrica $(m)$ y de la conductividad eléctrica (CE, $\mu \mathrm{S} / \mathrm{cm}$ ) registrada en JE5.

6. Irudia. JE5en erregistratutako kota piezometrikoen $(m)$ eta eroankortasun elektrikoaren (CE, $\mu \mathrm{S} / \mathrm{cm}$ ) aldaketa. 
Antes de iniciarse los bombeos hay aguas de características similares en JE9 y JE10 (CE en torno a 250 $\mu \mathrm{S} / \mathrm{cm}$ ), en JE2 y JE3 (sobre $220 \mu \mathrm{S} / \mathrm{cm}$ ), y en JE1 y DJH4 (175 $\mu \mathrm{S} / \mathrm{cm})$. Después de los bombeos se produce una aparente homogeneización de las aguas en los sondeos JE1, JE2 y DJH4, próximos entre sí, situándose la CE en valores cercanos a $200 \mu \mathrm{S} / \mathrm{cm}$. También tras los bombeos, destaca el aumento de CE en JE3 (ligeramente por encima de $250 \mu \mathrm{S} / \mathrm{cm}$ ), alcanzando valores similares a los que antes del bombeo tienen JE9 y JE10). Destaca, sobre todo, el ascenso de la CE que se produce en JE9, sobrepasando los $300 \mu \mathrm{S} / \mathrm{cm}$.

Estas observaciones hay que ponerlas en relación con las diferencias en los flujos de las aguas subterráneas antes y después de los bombeos. La homogeneización de las aguas en algunos sondeos, así como la aparición de aguas de mayor o menor mineralización en otros, indica la afección en cada sondeo de distintos niveles productivos.

\section{CONCLUSIONES}

Este artículo actualiza el conocimiento sobre la hidrogeología de la Masa de Agua de Jaizkibel, sobre todo a partir de la integración de diversas técnicas de investigación utilizadas a lo largo de 2012 en un estudio promocionado por la Diputación de Gipuzkoa. La Fig. 7 pretende mostrar el esquema conceptual de este conocimiento.

Se evidencia que estamos en presencia de un sistema multicapa: formaciones de areniscas, con un importante comportamiento acuífero, se alternan con formaciones, de naturaleza más lutítica, con un carácter acuitardo. El resultado es un conjunto de niveles productivos (acuíferos) más o menos individualizados por los acuitardos. La complejidad asociada a este tipo de sistema se manifiesta en las observaciones efectuadas en los sondeos. Así, los registros verticales (diagrafías) muestran diferencias en el tipo de agua presente en los pozos, e incluso en cada uno de ellos cuando son bom- beados. Los ensayos de bombeo realizados han permitido estimar los parámetros hidrogeológicos básicos: transmisividad $(T)$ entre 20 y $60 \mathrm{~m}^{2} / \mathrm{d}$, con heterogeneidad espacial y presencia puntual de efecto de barrera negativa, y coeficiente de almacenamiento (S) entre 0,004 y 0,009, propios de acuíferos confinados, o semi-confinados, acorde con el sistema multicapa.

El seguimiento piezométrico llevado a cabo en una importante red de pozos, evidencia una organización de los flujos subterráneos desde el SO de la vertiente norte del Jaizkibel hacia el NE, donde se localizan los manantiales más importantes. Los altos gradientes hidráulicos deducidos entre pozos cercanos hacen pensar en su relación a través de acuitardos. Ahora bien, los datos permiten distinguir claramente dos tipos de flujo: uno más profundo, observado en los pozos, con aguas más mineralizadas, y otro más somero, sub-superficial, que alimenta las numerosas regatas vertientes al mar. Los manantiales (Artzu, Justiz, Errota) parecen corresponder a zonas de descarga de esos dos flujos, si bien el más profundo debe de descargarse, en buena medida, directamente al mar. Más que la disposición de los estratos, buzantes hacia el NO, parece ser su vergencia y, quizás, la fracturación, la que facilita el sentido principal SO-NE.

El control continuo de niveles en un buen número de sondeos permite confirmar la falta de vías preferenciales de flujo subterráneo. La recarga de las aguas de precipitación se produce de forma lenta, difusa, sin que se observen variaciones significativas de nivel en esos momentos, aspecto éste acorde con el sistema multicapa. El hecho de que los flujos profundos sean de aguas más mineralizadas está indicando procesos de disolución del cemento calcáreo de los materiales detríticos, dando aguas de clara facies bicarbonatada cálcica. En contraposición, los flujos mas someros circulan por materiales mucho mas lavados, lo que justifica su menor mineralización y la mayor variabilidad de caudales observada en las regatas.

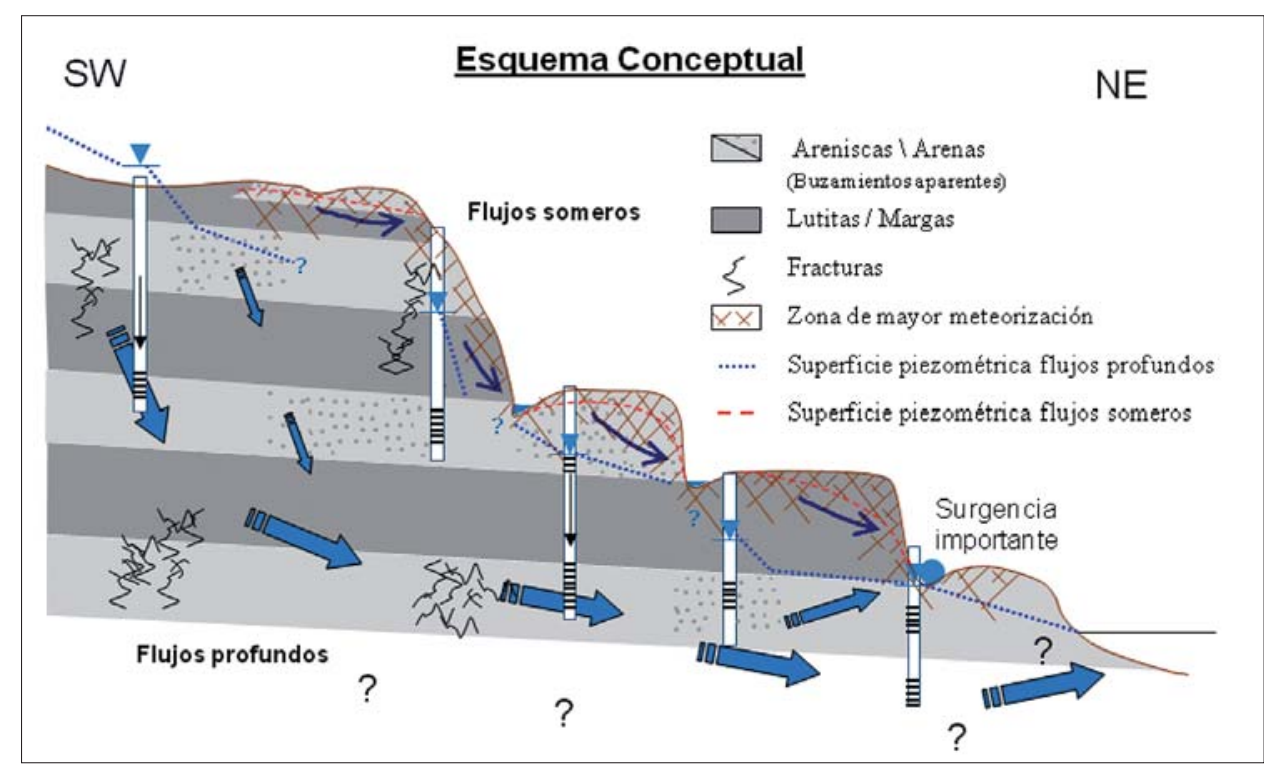

Fig. 7. - Esquema conceptual de funcionamiento hidrogeológico de la Masa de Agua Subterránea Jaikibel. 7. Irudia. Jaizkibel Lurrazpiko Ur Masaren funtzionamendu hidrogeologikoaren eskema kontzeptuala. 
Los ensayos de bombeo realizados, con caudales del orden de los $20 \mathrm{l} / \mathrm{s}$, sólo en algunos casos han mostrado influencia entre pozos cercanos, escasa en cualquier caso, con descensos de nivel en pozos de observación de unos pocos centímetros. Ahora bien, hay que señalar que estos ensayos han sido de muy corta duración (casi 1 día) como para poder evidenciar efectos que, en su caso, sólo en bombeos más prolongados llegarían a observarse. En cualquier caso, no se ha evidenciado afección algunas a los caudales de las regatas, ni de las surgencias, lo que puede explicarse por el hecho de que los bombeos afectan al flujo profundo, y no al somero.

Finalmente, aunque con este estudio se ha mejorado el conocimiento hidrogeológico de la Masa de Agua Jaizkibel, la infraestructura actualmente disponible (pozos, dispositivos de control) permite hacer un seguimiento más exhaustivo de los procesos hidrogeológicos en juego. Las técnicas utilizadas aportan claridad, pero requieren de series de datos más largas, y en distintas condiciones hidrológicas, incluyendo, sobre todo, el control de los bombeos, para poder extraer conclusiones que vayan completando el esquema conceptual.

\section{BIBLIOGRAFÍA}

Bredehoeft, J.D. 1967. Response of well-aquifer systems to Earth tides. J. Geophys. Res. 72: 3075-3087.

Diputación Foral de Gipuzkoa-Servicio Geológico del Ministerio de Obras Públicas-Euroestudios-Ingemisa S.A. 1986. Estudio de evaluación de los recursos hidráulicos subterráneos del Territorio $H^{\circ}$ de Gipuzkoa.

Diputación Foral de Gipuzkoa-Ente Vasco de la Energía (EVE)Geyser S.A.-Ingemisa S.A. 1993. Estudio Hidrogeológico de la cadena costera (Gipuzkoa).

Diputación Foral de Gipuzkoa-Hydrolur. 2011. Propuesta del perímetro de protección de las captaciones de Jaizkibel para el abastecimiento a la mancomunidad de aguas del Txingudi.

Diputación Foral de Gipuzkoa-Hydrolur. 2012. Mejora en el conocimiento Hidrogeológico de la masa de agua subterranea Jaizkibel = Jaizkibel lurpeko ur-masaren ezagutza hidrogeologikoaren hobekuntza.

Ente Vasco de la Energía-EVE. 1996. Mapa Hidrogeológico del País Vasco. Escala 1:100.000. 377 + Anexos.

Fernández de Ortega, I. 2007. Hidrogeología de las Sierras de Badaia y Arkamo (U. H. Calizas de Subijana, País Vasco). Tesis Doctoral. Universidad del País Vasco - Euskal Herriko Unibertsitatea.

Ferris, J.G. 1951. Cyclic fluctuations of water level as a basis for determining aquifer transmissibility. Int. Assoc. Sci. Hydrol. Publ. 33: 148-155. 